tion with catgut and section one-quarter of an inch be. low this; 6 , closure of the wound with a buried catgut stitch, and application of a collodion dressing. The entire operation can be performed on both sides in less than ten minutes, without any pain to the patient. The wound will heal so perfectly that no scar can be discovered.

The advantages of dealing with habitual criminals in this manner can be summed up as follows:

1. It would do away with hereditary criminals from the father's side.

2. Aside from being sterile the criminal would remain in his normal condition.

3. This method would protect the community at large without harming the criminal.

4. 'The same treatment could reasonably be suggested for chronic inebriates, imbeciles, perverts and paupers.

710 Sedgwick Street.

\title{
RECURRING GONORRHEA.
}

\section{BY HARRY GREENBERG, M.D.}

INSTRUCTOR IN GYNECOLOGY, WISCONSIN COLLEGE OF PHYSTCIANS AND SURGEONS. MILWALKEE, WIS.

V. F., single, merchant, aged 26 , consulted me on July 2, 1898 , for an attack of gonorrheal urethritis, and under treatment with potassium permanganate solution irrigations for one month, was discharged cured. He was then instructed to abstain from the use of alcohol in any form, and was warned against marrying for at least one year from date of discharge, as he was then engaged to be married to a refined young woman of this city.

On March 2, 1899, he again consulted me as to the advisability of marrying, and assured me that during all these months, he had noticed no discharge from his urethra, and at no time felt any inconvenience. He also stated - and I think truthfully-that he did not during that time partake of any alcoholic drinks, and had not indulged in sexual intercourse. Physical examination revealed nothing.

He was married one week later, and again consulted me in ten days from the date of his marriage, for a discharge from his urethra and all symptoms of acute gonorrheal urethritis, which diagnosis was substantiated by the microscope. Three days later his wife consulted me for burning urination and vaginal dis. charge, which on examination proved to be of gonorrheal infection, likewise substantiated by the microscope. This young woman I had treated for uterine displacement, and had examined her but two days previous to her marriage, and as $I$ at no time had occasion to doubt her innocence, the infection can be attributed to no other cause than that she contracted it from her husband.

This is but one of many similar cases that have come under my notice, but none, where I could so definitely attribute the recurring infection to an old and apparently cured gonorrhea.

This case is of interest in once more reviving the question: When may our patients, after cessation of the discharge from a gonorrheal urethritis, marry without fear of relapse and infecting the innocent woman? In an article in the Joursal of March 25, under the heading of "Treatment of Gonorrheal Urethritis," the author, after recommending a certain line of treatment, says that after the discharge ceases and micro-

scopic examination reveals no gonococci, the patient may be discharged and, in his own words, relapse is impossible without infection. The case above cited certainly does not warrant this, his positive assertion.

While it is true that many cases of gonorrheal urethritis suffer no relapse, it is als true, in many instances, that although the microscope will fail to show any gonococci in the urethral secretions, yet they exist latent in some of the folds of the urethra, harmless in their present state, but liable to regain their former virulence under favorable conditions, which a temporary congestion of the urethra will afford. Immoderate use of alcohol, and excessive venery-both conditions common in the newly-married -have the effect of producing a hyperemia of the sexual organs, and produce therefore a good nutrient medium for the latent gonococci to thrive on. It may also be possible that the gonococci, while harmless in their present position in the male urethra, after being deposited in the vagina, affording them a fresh soil, will again become virulent and produce a gonorrheal vaginitis, which will in return reinfect the man on a subsequent coition.

This same fact is also applicable in the female, after a gonorrheal vaginitis. I have observed, on examining prostitutes who had at some period been affected with gonorrhea, but were now apparently free from it, that they are nevertheless prone to infect the male uretbra with gonorrhea. I have also carefully investigated several cases where the man has contracted the infection from a woman who, before and after sexual intercourse with that man, was examined by me and no signs of gonorrhea could be detected. Evidently the latent gonococci in the folds of the vagina, after being deposited in the male urethra, began to thrive on the new soil and set up the inflammation.

545 E. Water St.

\section{RECURRENT RHEUMATIC IRITIS.*}

RECOVERY, WITH A SEGMENT OF THE IRIS FIXED WITHOUT APPARENT SYNECHIA, WITH NORMAL ACUITY

OF VISION AND WITHOUT INCREASE OF MYOPIA, IN PATIENT WITH MYOPIC ASTIGMATISM.

BY S. D. RISLEY, A.M., M.D.

Attending Surgeon Wills Eye Hospital; Professor of Diseases of the Eye, Philadelphia Polyclinic and College for Graduates in Medicine. PHILADELPHIA, PA.

Mr. A., aged 35 years, with well-marked rheumatic diathesis, has for several years been subject to recurring iritis of a severe type. I have treated him through three violent and persistent attacks and several milder exacerbations of the disease. The last and most violent came on as a simple hyperemia of the iris, with impaired visual acuity on Oct. 22, 1898. A full dilatation of the pupil was immediately secured, when it was discovered that the fundus presented a uniform fluffy appearance and that there was a fine exudate in the anterior portion of the vitreous. Notwithstanding active treatment addressed to his diathesis and to the local conditions, the eye developed a violent and painful attack of iritis of the parenchymatous type, which confined him to his room until the close of November, and he did not resume his business until the middle of January. The in-

* Read before the College of Physicians of Philadelphia-Section on Ophthalmology. 